\title{
The nature of rhyme processing in preliterate children
}

\author{
Barbara Wagensveld ${ }^{1,2, *}$, Petra van Alphen ${ }^{3}$, Eliane Segers' \\ and Ludo Verhoeven'
}

'Behavioural Science Institute, Radboud University Nijmegen, The Netherlands

${ }^{2}$ Donders Institute for Brain, Cognition and Behaviour, Centre for Cognitive

Neuroimaging, The Netherlands

${ }^{3}$ Max Planck Institute for Psycholinguistics, The Netherlands

Background. Rhyme awareness is one of the earliest forms of phonological awareness to develop and is assessed in many developmental studies by means of a simple rhyme task. The influence of more demanding experimental paradigms on rhyme judgment performance is often neglected. Addressing this issue may also shed light on whether rhyme processing is more global or analytical in nature.

Aims. The aim of the present study was to examine whether lexical status and global similarity relations influenced rhyme judgments in kindergarten children and if so, if there is an interaction between these two factors.

Sample. Participants were $4 \mathrm{I}$ monolingual Dutch-speaking preliterate kindergartners (average age 6.0 years) who had not yet received any formal reading education.

Method. To examine the effects of lexical status and phonological similarity processing, the kindergartners were asked to make rhyme judgements on (pseudo) word targets that rhymed, phonologically overlapped or were unrelated to (pseudo) word primes.

Results. Both a lexicality effect (pseudo-words were more difficult than words) and a global similarity effect (globally similar non-rhyming items were more difficult to reject than unrelated items) were observed. In addition, whereas in words the global similarity effect was only present in accuracy outcomes, in pseudo-words it was also observed in the response latencies. Furthermore, a large global similarity effect in pseudo-words correlated with a low score on short-term memory skills and grapheme knowledge.

Conclusions. Increasing task demands led to a more detailed assessment of rhyme processing skills. Current assessment paradigms should therefore be extended with more demanding conditions. In light of the views on rhyme processing, we propose that a combination of global and analytical strategies is used to make a correct rhyme judgment.

\footnotetext{
* Correspondence should be addressed to B. Wagensveld, Behavioural Science Institute, P. O. Box 9I04, 6500 HE Nijmegen, The Netherlands (e-mail: b.wagensveld@bsi.ru.nl).
} 


\section{The nature of rhyme processing in preliterate children}

The awareness of rhyme is one of the earliest forms of phonological awareness to develop (e.g., Vloedgraven \& Verhoeven, 2007), and is part of a unidimensional construct of phonological awareness which was found to be predictive of early reading skills in Dutch (de Jong \& van der Leij, 1999), as well as in other languages (e.g., Goswami, 2002). Therefore, rhyming tasks are an important component of phonological awareness assessments in both research and practice. In many of these assessments, rhyme awareness is measured by means of a simple rhyme decision task. For example, in the most commonly used preschool measure of phonological awareness in Dutch primary schools (CITO, 2009), children perform a rhyme task during which they are presented with three pictures representing the words buik (belly), kaal (bold), and vuur (fire) and are asked which of the words on the pictures rhymes with muur (wall). This can be considered as a fairly straightforward task since neither of the distracter words shares a relationship with the clue word; both are semantically and phonologically unrelated to the target. Furthermore, the children are presented with familiar words, which may make the processing of rhyme information easier than if they were presented with unfamiliar words.

This straightforward version of a rhyme decision task is also often used in the international research domain, despite the observations that more demanding experimental conditions (e.g., a condition with phonological distracters) can influence rhyme performance in young children (Cardoso-Martins, 1994; Carroll \& Snowling, 2001). Studies that did examine rhyming skills in a more demanding experimental setting did not always examine the explicit effect of higher task demands. In a study by Hulme and colleagues (2002), for example, rhyming skills were examined by using pseudo-words, but the absence of a control condition with words makes it difficult to examine to what extend rhyme performance was influenced by the artificial stimuli.

Straightforward rhyme tasks do not fully grasp the rhyming competence of children, and little is known about the direct influence of task enhancements on rhyme competence. The present study therefore addressed this issue in preliterate children in the Netherlands, and examined the influence of two task-enhancing factors. First, by presenting the same children with both words and pseudo-words, we tried to gain insight into the role of lexical representations in kindergartners' rhyme judgement. Second, we studied the effect of global phonological similarity in rhyme judgements in these children. A better understanding of the influence of these factors on rhyme processing can be used to improve the estimation of rhyme competence in phonological awareness assessments and develop better methods for phonological awareness instruction in preschool and Kindergarten.

\section{Rhyme processing: analytical or global?}

There are two diverging views on the process that underlies rhyme detection in young children: an analytical view and a global view. According to the analytical view, rhyme judgements are based on an explicit phonological process during which children consciously segment the rime constituent of the first word and compare it to the rime counterpart of the second word to make a judgement (Bryant \& Bradley, 1985; Bryant, MacLean, Bradley, \& Crossland, 1990; Kirtley, Bryant, MacLean, \& Bradley, 1989; Ziegler \& Goswami, 2005). This analytical view is supported by the onset-rime theory (Treiman \& Kessler, 1995; Ziegler \& Goswami, 2005). According to this theory syllables in languages such as Dutch and English have a natural breaking point dividing the 
syllable into an onset, which consists of the first consonant (cluster), and a rime, which encompasses the vowel and final consonant (cluster). The available rime structures can be used to make an explicit analytical decision during a rhyme judgement task.

Although the onset-rime theory is widely accepted, there is only little evidence that children, especially young preliterate children, find rhyme judgement tasks more difficult when the task demands are increased. One study by Cardoso-Martins (1994) showed that young children who perform well on a simple rhyme judgement task get confused when they are presented with phonologically related distracters that do not rhyme. She first presented Brazilian preschool, kindergarten and first-grade children with a simple rhymecategorization task with semantic distracters. Children who were able to perform above chance level on this fairly simple task were presented with a second version of the task in which the semantic distracters were replaced by phonologically related items. This second task was more difficult for the preliterate children; only 10\% of the preschoolers and $43 \%$ of all kindergartners performed above chance level in comparison to $83 \%$ of the first graders with reading experience. These results point to a more global approach in the making of rhyme decisions by especially preschool and kindergarten children.

Carroll and Snowling (2001) also found evidence that rhyme judgements are based on the comparison of global phonological similarities. In their study, English preschoolers performed a picture rhyming task. The children were presented with a clue picture and were asked to select the rhyming target from two alternatives; one rhymed with the clue word and the other was a distracter. There were three types of distracters; one distracter was unrelated to the clue word (e.g., dish: fish-pen), one was phonologically related to the clue word (e.g., top: mop-tap) and one was semantically related to the clue word (e.g., cat: hat-dog). Results showed that although the children performed above chance level on all three conditions, the items that were most similar on a global level, namely the phonologically related items, were more difficult to reject than the other distracters.

These observations of this so-called global similarity effect make it difficult to explain rhyme judgement solely by the analytic onset-rime theory. When children are presented with a more demanding condition, containing phonologically overlapping items, their proficiency decreases. These findings have led to an alternative theory that states that preliterate children use a more implicit approach to judge rhyme overlap in words that is based on a global comparison of a word's phonological structure.

\section{The role of lexical representations in rhyme processing}

An explanation for the global similarity effect has been sought in the ill-defined nature of the underlying lexical representations. Theories on the development of the mental lexicon state that the lexicon of young children mainly contains holistic representations as opposed to fully specified segmental units, that is, words are stored as a holistic unit and onset-rime information will be less available in young children (Charles-Luce \& Luce, 1990; Jusczyk, 1993; Metsala \& Walley, 1998; Walley, 1993; Walley, Metsala, \& Garlock, 2003).

Carroll and Snowling (2001) proposed that these holistic representations make it difficult to perform well on a rhyme task with phonological distracters. For example, if young children are presented with a prime word (e.g., top) they have great difficulty choosing between the rhyming target (e.g., mop) and the globally similar target (e.g., tap), since both targets share two common phonemes with the prime. They are perceived as equally similar to the prime item due to the holistic nature of their representations. 
Alternatively, there is evidence that phonological representations of children are segmental from an earlier age on. A Dutch recall study showed that 2-year-olds made phoneme substitutions when they were asked to repeat syllables (Wijnen, 1992). These substitutions at the segmental level provide evidence that there are at least partially segmented representations available in 2-year-olds. This observation makes it difficult to explain the global similarity effect in terms of ill-defined representations. Furthermore, it questions the implicit nature of rhyme decisions that was suggested by Carroll and Snowling (2001) and again provides evidence in favour of the onset-rime theory which describes a more explicit phonological process.

Up till now, the hypothesis that holistic representations lie at the basis of the global similarity effect has been explored by making use of the phonological neigbbourbood density effect ${ }^{1}$ (De Cara \& Goswami, 2003; Stadler, Watson, \& Skahan, 2007) using the rationale that the lexical representations in dense phonological neighbourhoods will be more specifically defined than those from a sparse neighbourhood, since they need to be distinguished from the many other items in that neighbourhood. However, studies using this rationale have found contradicting results on the role of lexical representations in the global similarity processing (De Cara \& Goswami, 2003; Stadler et al., 2007).

Given these contradictory findings, the present study followed an alternative approach by examining pseudo-words in addition to words, making it possible to examine rhyming skills with unfamiliar items that are not represented in the lexicon. Pseudowords are phonologically legal combinations of phonemes that do not form an existing word (e.g., nool). These words and their constituent onset-rime structures can be considered as not stored in the mental lexicon. In previous studies that examined the role of global similarity in rhyme judgements, the children were only presented with word stimuli (Carroll \& Snowling, 2001). Although pseudo-words are used in studies that examine rhyme performance (Hulme et al., 2002), the pseudo-words in those studies were not contrasted with a word condition, making it unclear how this kind of task enhancement influences the results. To the best of our knowledge, there are no studies that directly contrasted preliterates rhyme judgements of words and pseudo-words with global similarity as an experimental condition. Since pseudo-words are not stored in the mental lexicon, the use of these stimuli can provide information on the role of lexical representations during rhyme judgements.

\section{The present study}

In the present study, we presented Dutch children with a word and pseudo-word version of the same rhyme judgement task containing phonologically related nonrhyming distracters. This paradigm enabled us to examine the influence of both lexicality and global similarity on rhyme judgements in more detail. Specifically, by using this paradigm we were able to investigate in what way lexical representations influence rhyme processing in children. Dutch phonology offers a great variety of short consonantvowel-consonant (CVC) words, making it relatively easy to find minimal CVC word pairs (Nunn, 1998) and to derive similar pairs of pseudo-words.

Following an experimental approach, children in Kindergarten were asked to make rhyme judgements on auditory pairs in three phonological conditions; one rhyming

\footnotetext{
I A phonological neighbourhood contains all words that differ from each other by only one phoneme, for example, mop and tap are both phonological neighbours of top but not from one another.
} 
condition (e.g., kus-mus) and two non-rhyming conditions. The non-rhyming conditions contained either globally similar items (e.g., mes-mus) or unrelated items (e.g., bak$m u s)^{2}$. The children were presented with both words and pseudo-words in two separate blocks. In addition to accuracy, we also measured response times of children's rhyme judgements in the present study as an index of processing difficulty. To examine whether rhyming is a more analytical, memory-dependent process, the children were also presented with two memory tasks: a pseudo-word repetition task and a word span task. And, since grapheme knowledge is an indicator of more advanced phonological awareness (Johnston, Anderson, \& Holligon, 1996), it may also be an indicator of using a more analytical approach in rhyme judgements. The children were therefore also presented with a grapheme knowledge task.

The main question of the present study consisted of three parts. We examined whether lexical status (1) and global similarity relations (2) influence rhyme judgments in Kindergarten children and if so, if there is an interaction between these two factors (3). In addition, we wanted to gain insight into the relationship between the two factors and memory and letter knowledge skills.

\section{Method}

\section{Participants}

Participants were 41 monolingual Dutch-speaking children (21 girls, 20 boys) from two schools in the middle part of the Netherlands. All children were tested during their second year of kindergarten (which is a 2-year program in the Netherlands) and were on average 6.0-years old ( 72 months, $S D$ 4.3). Children had not received any formal reading education; this starts in first grade in the Netherlands. Prior to participation, the children's parents filled out a questionnaire. Only those children that were right handed, had normal hearing and (corrected to) normal sight and no known history of neurological problems were selected to take part in the experiment. The study was approved by the national ethics committee and the parents of all participants signed informed consent.

\section{Materials}

Memory skills

Verbal memory skills were assessed in two tests: a word span task and a pseudoword repetition task. Both tasks are subtests of the screening instrument for severe speech and language problems in Dutch (Verhoeven, 2011). The word span task contained 12 sequences of monosyllabic Dutch words, which were spoken out loud by the experimenter. Sequences varied in length from two to seven words. The children were asked to repeat sequences of monosyllabic Dutch words spoken out loud by the experimenter. Responses were scored as either correct or incorrect and the test was ended if a child was unable to repeat four consecutive sequences. The stimuli of the pseudoword repetition task were 40 pseudo-words, which were spoken out loud by the experimenter. The pseudo-words varied in length from one to six syllables. During the task, the child was asked to repeat a pseudo-word spoken out loud by the experimenter.

\footnotetext{
2 In this study, we used a prime-target rhyme judgment approach instead of more commonly used rhyme tasks such as the oddity task (Bradley \& Bryant, 1983) in order to take accurate response time measures.
} 
Table I. Mean frequency and duration of stimuli used in the rhyme judgement task. Mean frequencies are presented in percentages, which are calculated using the 'Streeflijst woordenschat voor zesjarigen', an index of vocabulary knowledge of Dutch-speaking 6-year-old children. Mean durations are presented in milliseconds. Standard deviations of the mean are in parentheses

\begin{tabular}{lccc}
\hline & $\begin{array}{c}\text { Mean frequency } \\
\text { Words }\end{array}$ & $\begin{array}{c}\text { Mean duration } \\
\text { Words }\end{array}$ & Pseudowords \\
\hline Rhyming primes & $96(6)$ & $498(85)$ & $511(83)$ \\
Overlapping primes & $96(5)$ & $508(89)$ & $515(93)$ \\
Unrelated primes & $98(4)$ & $477(108)$ & $490(85)$ \\
Targets & $97(4)$ & $506(84)$ & $492(58)$ \\
Filler primes & $97(4)$ & $528(92)$ & $506(80)$ \\
Filler targets & $97(3)$ & $525(93)$ & $478(66)$ \\
\hline
\end{tabular}

Responses were scored as either correct or incorrect. The task was ended if a child failed to repeat five consecutive pseudo-words.

\section{Grapheme knowledge}

Grapheme knowledge was assessed by presenting the children with a chart containing all 34 Dutch graphemes. The children were asked to name all graphemes that were familiar to them out loud. Responses were scored as either correct or incorrect. This task was a subtest of the ESM test, a Dutch screening test for language and reading problems (Verhoeven, 2008).

\section{Rhyme judgement}

The stimuli of the rhyme judgement experiment consisted of a set of monosyllabic words and pseudo-words with a CVC structure. Words were selected from the 'Streeflijst Woordenschat voor Zesjarigen' (Schaerlaekens, Kohnstamm, \& Lejaegere, 1999), an index of vocabulary knowledge of 6-year-old Dutch-speaking children. This list provides all Dutch words with the percentage of Dutch and Belgian teachers who expect 6-yearold children to understand the word. All selected words were indexed above $80 \%$. The average percentages of the words can be found in Table 1 . Stimuli were spoken by a female speaker and digitally recorded $(44.1 \mathrm{kHz}$, stereo) in a sound attenuated room using a Sennheiser ME62 microphone connected to a Dell D610 latitude laptop running Sony Sound Forge, a sound recording program. After recording, each stimulus was carefully edited for precise onset and offset using a speech waveform editor (Praat, version 4.5.12). Mean durations of the targets and the rhyming, overlapping, and unrelated primes can be found in Table 1 .

Word condition. Word stimuli consisted of 21 target words (e.g., bek or boek). Each target was paired with three different types of prime words, resulting in three conditions. In the rbyming condition, the target was paired with a prime that shared the rime constituent of the word (e.g., gek-bek or koek-boek). In the unrelated condition, the target was paired with a prime word that did not share any phonological overlap with the target (e.g., sop-bek or nies-boek). In the overlap condition, the target word was paired with a prime word that shared a phonological overlap with the target word, but 
did not rhyme. In this overlap condition, $50 \%$ of the trials contained a consonant overlap (e.g., bak-bek) and 50\% contained a vowel overlap (e.g., poes-boek). In the overlap condition, each target was only presented once per participant, thus either with a prime that overlapped in consonant or a prime that overlapped in vowel. The presentation of these primes was counterbalanced over participants. A list of all the word stimuli and translations of the words can be found in the Appendix, Table A1.

Pseudo-word condition. Pseudo-word targets were created by re-associating the onset of one word target with the rime of another word target to control for bigram frequencies in words and pseudo-words. This resulted in 21 monosyllabic phonologically legal Dutch pseudo-word targets that consisted of exactly the same phonemes as the targets in the word condition. The same recombination method was used to create 63 pseudo-word primes from the word primes in the three phonological conditions. This resulted in the prime target combinations; rhyming pairs (e.g., baam-daam or mip-bip), unrelated pairs (e.g., not-daam or gos-bip), and overlap pairs (e.g., diem-daam or mit-bip). A list of all the pseudo-word stimuli can be found in the Appendix, Table A2.

Fillers. Because there was only one rhyming condition and two non-rhyming conditions, it was likely that children would develop a response bias towards the non-rhyming conditions. To prevent this, 21 monosyllabic word and pseudo-word filler pairs were created, which served as fillers in the experiment and were excluded from further analysis. A list of all the filler stimuli and translations of the filler words can be found in the Appendix, Table A3.

Similarity factor. To be able to compare the effect of global similarity in the word and pseudo-word condition, the degree of global similarity in the two conditions needs to be the same. To make sure that the amount of global similarity in the word and pseudo-word overlap condition was equal, we calculated a similarity factor (SF). The SF was computed using the Dutch diphone database from a study by Smits, Warner, McQueen, \& Cutler (2003). In this gating study, adult Dutch participants were asked to identify the Dutch diphones. Responses to the diphones were used to create a confusion matrix, indicating the likelihood of a phoneme being correctly interpreted as itself or as any of the other phonemes. In the present study, these values were used as an indicator of phonological similarity amongst pairs. Mean global similarity values were computed per condition and can be found in Table 2. Please note that the higher the score is, the more similar a pair is.

\section{Procedure}

Prior to the rhyming experiment, we assessed the children's verbal short-term memory in word span task and a pseudo-word repetition task in a separate session. Each child was tested in a quiet room in the school.

In a second session, the rhyming task was assessed. Before the actual test began, the experimenter held a short practice interview with the child, in which the child was asked to think of a matching rhyme word of a word provided by the experimenter. If the child could not produce a rhyme word, an example was given and the child was encouraged 
Table 2. Mean phonological similarity of word and pseudo-word pairs per condition. The phonological similarity is based on data from the Dutch diphone database (Smits et al., 2003). The higher the global similarity value, the more alike the pairs are. Standard deviations of the means are in parentheses

\begin{tabular}{lcccc}
\hline & \multicolumn{3}{c}{ Phonological similarity } \\
\cline { 2 - 5 } & \multicolumn{3}{c}{ Words } & \multicolumn{2}{c}{ Pseudowords } \\
\hline Rhyming pairs & 1,620 & $(371)$ & 1,610 & $(399)$ \\
Overlapping pairs & 1,014 & $(335)$ & 1,003 & $(309)$ \\
Unrelated pairs & 33 & $(47)$ & 43 & $(47)$ \\
\hline
\end{tabular}

to think of another example. After this short practice, the child was presented with the experimental task.

The experimental task was divided into two separate parts with a break in between. In the first part, the children were presented with the words and in the second part they were presented with the pseudo-words ${ }^{3}$. Both the word and pseudo-word part began with a short training block in which the child was presented with six (pseudo) word pairs, two of each condition. After the training block, there were three experimental blocks. The three conditions were divided evenly over these blocks and each target was presented once per block with one of the prime conditions (rhyme, unrelated, or overlap) resulting in 21 trials per block and 63 experimental trials in total. The presentation order of blocks was counterbalanced across participants. There was a break between blocks, the duration of which depended on the need of the child.

During the rhyme judgement experiment, stimuli were presented binaurally using Presentation 9.70 over a Sennheiser HD 433 headphone (Wedemark, Germany) at a comfortable listening level of $65 \mathrm{~dB}$. The child was instructed to indicate with a button press whether two members of a pair that were presented over headphones rhymed or not. Response hands for rhyme and non-rhyme were counterbalanced over participants. Each trial began with the presentation of a fixation cross in the middle of the screen for $950 \mathrm{~ms}$. Fifty millisecond later the prime word was pronounced and $1200 \mathrm{~ms}$ after the prime onset the target word was pronounced. These words were not presented on-screen, only through the headphones.

\section{Results}

\section{Descriptives}

The mean response latency of the correct responses to the targets and the percentage of incorrect responses to the targets of each of the three phonological conditions were calculated per subject. Response times were measured from the onset of the target. Table 3 displays the mean response times and error scores of the kindergarten children on average and for each of the three phonological conditions separately for words and pseudo-words.

The two memory tasks had the following outcomes. In the pseudo-word repetition task, children named between 17 and 38 items correct resulting in an average score of

3 Children were always presented with the easier word version of the task first. Following this approach, the children were able to adapt to the task and built up their confidence for the more difficult pseudo-word version of the task. 
Table 3. Mean response latencies and accuracy scores on the rhyme judgement task. Response latencies are presented in milliseconds and error scores are presented in the percentage of incorrect answers per condition. Standard deviations of the means are in parentheses

\begin{tabular}{|c|c|c|c|c|c|c|c|c|}
\hline \multirow[b]{3}{*}{ Average } & \multicolumn{4}{|c|}{ Response latency } & \multicolumn{4}{|c|}{ Error score } \\
\hline & \multicolumn{2}{|c|}{ Words } & \multicolumn{2}{|c|}{ Pseudowords } & \multicolumn{2}{|c|}{ Words } & \multicolumn{2}{|c|}{ Pseudowords } \\
\hline & $|, 84|$ & $(304)$ & $1,99 \mid$ & (404) & 10.4 & $(10.1)$ & 18.1 & $(14.6)$ \\
\hline Rhyming pairs & 1,719 & (334) & 1,952 & (435) & 6.9 & $(9.3)$ & 13.8 & $(15.1)$ \\
\hline Overlapping pairs & 1,937 & (345) & 2,050 & (445) & 15.4 & (I3.7) & 23.1 & $(21.9)$ \\
\hline Unrelated pairs & $\mathrm{I}, 867$ & (345) & 1,972 & (408) & 9.0 & $(12.3)$ & 17.5 & (19.4) \\
\hline
\end{tabular}

28.59 ( $S D$ 4.96). In the word span task, children were able to correctly repeat between two and eight sequences. The average score on the word span task was 4.54 (SD 1.19). Correct identifications of the graphemes in the grapheme knowledge task varied between one and 29 items correct. The average score was 14.22 (SD 7.34).

\section{Statistical analysis}

Two repeated measures analyses of variance were undertaken with lexical status (word, pseudo-word) and phonological condition (rhyme, overlap ${ }^{4}$, unrelated) as within subject factors, for both error score and response latency.

\section{Lexical effects}

Main effects of lexical status were found in both response time and accuracy data $\left(F_{r t}(1,40)=14.07, p<.001, \eta^{2}{ }_{p}=.26 ; F_{a c c}(1,40)=20.64, p<.001, \eta^{2}=.34\right)$. These findings showed that in all three phonological conditions children responded slower to pseudo-words (1991 ms, SD 404) than to words (1841 ms, SD 304). Furthermore, children made more errors in the pseudo-word condition (18.1, $S D$ 14.6) than the word condition (10.4, SD 10.1). The general pattern of both response times and accuracy scores showed that Dutch kindergartners experienced more difficulties in pseudo-word rhyming than in word rhyming.

\section{Global similarity effects}

Main effects of phonological condition were observed in both the response latency and accuracy outcomes $\left(F_{r t}(2,39)=13.16, p<.001, \eta_{p}^{2}=.40 ; F_{a c c}(2,39)=\right.$ 9.14, $\left.p<.001, \eta_{p}^{2}=.34\right)$. The significant main effects for phonological condition were further investigated by performing paired sample $t$-tests on the following three planned comparisons; overlap versus unrelated, overlap versus rhyme, and rhyme versus unrelated. Of special interest to us were any differences between the overlapping and unrelated condition. Both of these conditions should be judged as non-rhyming. If children were judging word pairs on the basis of global similarity relations, we would expect to see slower response times and lower accuracy scores for the overlap conditions

\footnotetext{
4 No differences were observed between pairs overlapping in consonants and pairs overlapping in vowel. Therefore, these two conditions were collapsed and referred to as the overlap condition.
} 
(e.g., mes-mus) as compared with the unrelated condition (e.g., bak-mus). Outcomes showed that overlapping pairs were judged more slowly and less accurate than both unrelated $\left(t_{\mathrm{rt}}(40)=2.73, p<.01, d=.19 ; t_{\mathrm{acc}}(40)=3.85, p<.001, d=.35\right)$ and rhyming pairs $\left(t_{\mathrm{rt}}(40)=5.18, p<.001, d=.41 ; t_{\mathrm{acc}}(40)=3.62, p<.005, d=.59\right)$. Furthermore, rhyming pairs were judged faster than unrelated pairs $(t(40)=-2.60, p<$ $.05, d=.22$ ), however, we could not observe any differences in accuracy between the rhyming and unrelated condition.

\section{Interactions between lexicality and global similarity}

An interaction between lexical status and global similarity relations was only found in response time measures not in the accuracy scores $\left(F_{r t}(2,39)=4.24, p<.022, \eta^{2} p=\right.$ .18; $\left(F_{a c c}(2,39)<1\right)$. The observed interaction between lexical status and phonological condition was further examined by conducting pair-wise comparisons. Outcomes showed that the response latencies of word and pseudo-word rhyme judgements showed two distinct patterns. In word judgements, rhyming pairs were judged faster than both overlapping $(t(40)=-5.02, p<.001, d=.65)$ and unrelated pairs $(t(40)=-3.84$, $p<.001, d=.44)$ whereas no differences could be observed between overlapping and unrelated pairs. In pseudo-word judgements, both rhyming $(t(40)=-2.27, p<.05$, $d=.23)$ and unrelated pairs $(t(40)=-2.29, p<.05, d=.18)$ were judged faster than overlapping pairs, and no differences were observed between rhyming and unrelated pairs.

In sum, a global similarity effect was observed in the accuracy outcomes of both word and pseudo-word rhyming. In the response time data, the global similarity effect was only observed in pseudo-words not words.

\section{Correlations with memory skills and grapheme knowledge}

The relationship between short-term memory skills, grapheme knowledge, and effects of phonological similarity on the rhyme judgment performance was examined by performing one-tailed bivariate Pearson's correlations. The effect of phonological overlap, that is, the global similarity effect, was computed by subtracting the mean response latency to unrelated pairs from the mean response latency to overlapping pairs. Accuracy effects were computed by subtracting error scores in a similar fashion. Differences were calculated for words and pseudo-words separately resulting in four values per participant, which were correlated to the scores on the grapheme knowledge and verbal short-term memory tasks.

The score on the word span task correlated significantly with the overlap effect in response latency to pseudo-words $(r=-.38, p<.05)$. In addition, a trend was observed for pseudo-word repetition and the overlap effect in response latency to pseudo-words $(r=-.26, p<.06)$. Both outcomes indicate that the higher the memory score, the smaller the global similarity effect. Furthermore, the score on the grapheme knowledge task correlated with the global similarity effect in accuracy score in the pseudo-word condition $(r=-.27, p<.05)$, which indicates that the more graphemes the children know, the smaller the difference is in the percentage of errors between overlapping and unrelated pseudo-word items. There were no significant correlations between the global similarity effect in words and the short-term memory tasks or grapheme knowledge. 


\section{Discussion and Conclusion}

The present study examined the effects of two types of task enhancement on rhyme judgement skills of Dutch kindergarten children. Specifically, we were interested in the influence of lexicality and global similarity on rhyme judgement skills. The kindergarten children performed quite well on the rhyme judgement task. Accuracy scores on all phonological conditions were well above chance level ( $76 \%$ or more correct), indicating that the children were able to correctly judge whether two members of a pair rhymed or not. However, although children were able to carry out the task they showed difficulties when the task became more demanding due to the use of non-lexical or globally similar items.

\section{Lexicality effects in rhyme judgment}

Since most rhyme assessments make use of either word or pseudo-word stimuli, the first part of our question was whether or not there are differences in rhyming with words or pseudo-words. We observed that enhancing the task by presenting pseudo-words led to poorer performance than when children are presented with familiar words; they made more errors and responded slower to items that were not represented in the mental lexicon ${ }^{5}$. This finding might not be surprising as lexicality effects are observed in many tasks especially with young children (Jarrold, Cocksey, \& Dockerill, 2008; Zoccolotti, De Luca, Di Filippo, Judica, \& Martelli, 2009), However, as has been said, the influence of lexicality is often neglected in rhyme judgment assessments in both research and practice. Most studies examined rhyme judgment skills by using only word (e.g., Cardoso-Martins, 1994; Carroll \& Snowling, 2001) or pseudo-word stimuli (e.g., Hulme et al., 2002). The outcomes of these studies seem to provide a unilateral view on the rhyme performance of children. On the one hand, studies examining word rhyming may not be challenging enough even for young children. On the other hand, presenting children with only pseudo-words may be too difficult for some children leading to the idea that they are not proficient at rhyming yet and furthermore, the use of pseudo-words may also influence other conditions of interest (such as the global similarity condition in the present study). In light of the lexicality effect observed in this study, it seems that both word and pseudo-word stimuli are needed to gain insight in the developmental pattern of rhyme awareness in children.

\section{Global similarity relations in rhyme judgment}

The influence of including more demanding conditions in rhyme judgment assessments was further explored in the second question of our study. We examined whether rhyme judgement performance was influenced by global similarities within non-rhyming pairs. The kindergarten children in the present study showed a clear global similarity effect when we compared responses to overlapping and unrelated items. They found phonologically overlapping non-rhyming pairs difficult to judge as was indicated by slower response times and higher error scores for these pairs as compared to phonologically unrelated non-rhyming pairs. The observation of a main effect of global similarity

\footnotetext{
5 For the reaction times, there was a prominent lexical status effect in the rhyming condition, but this effect was less clear in the overlap and unrelated condition. These outcomes could be the result of difference in complexity of the responses. The children had to respond 'yes' when a pair rhymed, and 'no' when a pair was either unrelated or overlapping. Especially with these young children, it is not unlikely that they found it more complex to give a 'no' answer than a 'yes' answer.
} 
indicated that global similarity relations played a role in both word and pseudo-word rhyming. But the observed interaction between lexical status and global similarity in the response time data indicated that there are differences in the behavioural response pattern to words and pseudo-words.

In word rhyming, children made more errors in the globally similar overlap condition than in the unrelated non-rhyming condition. These observations are in line with findings in other studies that examined global similarity in rhyme processing in young children. Cardoso-Martins (1994) and Carroll and Snowling (2001) have shown that, although preliterate children could easily judge rhyming and unrelated word pairs, they made more errors when judging the phonologically similar, non-rhyming items. The present study extended these findings by examining global similarity effects in pseudo-words in addition to words. The results showed that, similar to words, the children made more errors when they judged non-rhyming pairs that were globally similar as compared to unrelated. In addition, they also responded slower to globally similar pseudo-word pairs as compared to unrelated pairs, an effect that could not be observed in word rhyming. This finding indicated that children have more difficulties with global similarity relations in pseudo-word rhyming than word rhyming.

Additional differences between word and pseudo-word rhyming were observed in the correlation analyses. We found correlations between short-term memory skills, grapheme knowledge, and pseudo-words rhyming. First, children with high verbal short-term memory scores were less sensitive to phonological overlap in pseudo-word pairs. As for correlations between grapheme knowledge and global similarity effect, we found that the more graphemes a child knows the smaller the global similarity effect is in the accuracy scores of the pseudo-words condition. No significant correlations were found with the global similarity effect in words.

The differences between the global similarity effect in word and pseudo-word rhyming again provide evidence that the use of task-enhancing factors should be carefully controlled and that using only familiar stimuli may not provide enough detailed information on children's ability to judge rhyme.

\section{Theoretical implications}

So far, the process that underlies rhyme judgments of young children has been explained by two diverging theories. In the first theory, an explanation for the global similarity effect has been sought in the nature of young children's lexical representations (Carroll and Snowling, 2001). This suggestion was based on the idea that the stored lexical representations of young children are rather holistic (Charles-Luce \& Luce, 1990; Jusczyk, 1993; Walley et al., 2003). The alternate view encompasses the onset-rime theory and states that rhyme judging is an analytical process that relies on children's ability to analytically compare rime constituent of (pseudo) words.

The present study did not specifically contrast these hypotheses, but the current data do provide some insights into this matter. Since global similarity relations were examined by using pseudo-words that are not (fully) represented in the mental lexicon, we were able to examine the contribution of the lexicon. The observation that words are processed faster than pseudo-words does indicate that support from the metal lexicon facilitates the making of rhyme judgement. In other words, having a lexical representation (even though this representation may not yet been fully specified) makes it easier to compare two items at a global level. However, the present data are not completely consistent with the lexical representations hypothesis, since children did not 
only show a global similarity effect in word rhyming but also in pseudo-word rhyming. This indicates that global similarity relations also influence rhyme judgment on items that are not represented in the lexicon, and therefore the nature of lexical cannot be considered as the sole cause of the global similarity effect.

Pseudo-word rhyming seems to be more analytical in nature. For example, the outcomes of the correlation analyses showed that pseudo-word rhyming depends on temporary phonological representations rather than stored representations in the mental lexicon. The absence of significant correlations between the verbal short-term memory tasks, grapheme knowledge and word rhyming and the observation of a global similarity effect in word rhyming indicate that children make use of a more global approach in word rhyming. It is important to note that analytical processes do play a role in word rhyming, as children were able to correctly discard the unrelated items.

In sum, the present findings suggest that rhyme judgements are not purely based on global phonological similarities nor are they solely the result of an analytical processing. Instead, we propose that a combination of these two theories explains how young children make rhyme judgements. The present and previous studies on global similarity processing have shown that children have an initial tendency to make a global comparison that is often enough for a correct rhyme judgement. We propose that this tendency is the result of an innate sensitivity to detect similarities in any kind of perceived information. This tendency has, for example, been observed in studies that examine phonological sensitivities in newborns (see Kuhl, 2004 for a review). For rhyme, studies have shown that even 9-month-old infants can detect a change in rhyme pattern or even smaller changes within the rhyme constituent of a word (Hayes, Slater, \& Brown, 2000; Hayes, Slater, \& Longmore, 2009). We carefully propose that this sensitivity and not the representations in the mental lexicon lie at the basis of the global similarity effect. We suggest that if children are presented with items that sound similar there is an immediate neural response to the overlap in the phonological patterns. This response can be used in a simple version of a rhyme task in order to make correct decisions since the similar sounding pair requires a yes response and the non-similar sounding pair requires a no response. The situation becomes more complex when children are presented with similar sounding pairs that do require a no response, as the overlap condition in the present study. The global similarity approach does not longer suffice and needs to be overruled by a more analytical approach as has been proposed by the onset-rime theory. Data from the present study support this idea since children were able to perform well on the overlap condition (more than $76 \%$ of the answers were correct). However, the innate sensitivity to phonological similarities does lead to longer response times and more often erroneous answers in the overlap condition.

Age and possibly reading experience could have influence on our assumption that rhyme judgements are initially based on globally similar characteristics in and become more analytical when needed. In two phonological classification experiments, Treiman and colleagues (Treiman \& Baron, 1981; Treiman \& Breaux, 1982) showed that preliterate children base their classifications on global similarity relations between lexical items whereas adult processing relies primarily on common phoneme relations. A more recent study confirmed the observation that phonemic information in word classification becomes more available with age (Carroll \& Myers, 2010). In order to use an analytical approach, children must be fully aware of the phonological structure of the prime and target. Children in kindergarten are in the process of becoming phonological aware, but they have not fully mastered this yet. Studies by Geudens and colleagues (Geudens \& Sandra, 2003; Geudens, Sandra, \& Martensen, 2005) have questioned the availability of 
onset-rime structures in Dutch-speaking pre-readers. In their studies, they could only obtain evidence for an onset-rime structure using an implicit similarity judgement task. All tasks tapping the more explicit forms of phonological awareness failed to show onsetrime effects. They interpreted their findings as evidence that the sensitivity of pre-readers to rhyme depends on similarity relations rather than explicit knowledge of the rime unit.

\section{Limitations and suggestions for future research}

One interesting question is what would happen when children are more experienced in phonological processing. The analytical approach that is presupposed by the onset-rime theory demands a full awareness of the phonological structure of the test items - a stage of phonological awareness, which does not develop until formal reading education has started (Goswami \& Bryant, 1990). The outcomes of the grapheme knowledge task in the present study may be carefully considered as an indication that literacy experience can lead to a more analytical approach, even for words.

Follow-up studies can thus be recommended to examine the performance of literate children in rhyme judgement in both words and pseudo-words. Outcomes of these studies may even contribute to the ongoing debate about whether or not rhyme is predictive of later reading skills (Bryant et al., 1990; Goswami, 1999; Hulme et al., 2002; Macmillan, 2002; Stuart, 2005). As has been said, the current findings indicate that simple rhyme judgement tasks might not be sensitive enough to measure rhyme awareness skills in detail. If rhyme awareness tasks would be more demanding, it is possible that the outcomes provide a better or clearer indication of later reading skills.

Another suggestion for follow-up research would be to examine if short-term memory does play a role when multi-syllabic words are used in rhyme judgement tasks, as it can be expected that short-term memory will then affect the judgement scores of words as well. Also, it would be interesting to examine to which extend sub-lexical information, such as phonotactic probability, influences the rhyme judgement performance with pseudowords. And, to examine the overlap effect in more detail, it would be interesting to examine a condition that shares an onset overlap (e.g., bes-bek). One shortcoming of the present study is that we did not take a vocabulary measure, which would have allowed us to directly relate the expanding lexicon to the rhyme judgement performances. Including such measures would be a final suggestion for future research.

\section{Conclusions}

To conclude, the general aim of the present study was to examine the influence of task enhancement on rhyme judgement skills of Dutch kindergarten children. The outcomes of the rhyme judgement experiment show that, although the children performed well on a rhyme judgement task, their proficiency decreased when we introduced non-lexical or globally similar items. We proposed that rhyme processing in preliterate children is based on an innate tendency to process information on the basis of global similarity relations but that this tendency can be overruled when an analytical rhyme decision is required due to higher task demands. As for practical implications, the present data show that higher task demands provide better insight in the development of rhyme processing. Therefore, it is suggested that more challenging conditions (i.e., pseudowords and globally similar pairs) are included to be able to measure rhyme awareness in more detail. 


\section{Acknowledgements}

The authors would like to thank all the children, their schools, and teachers and their parents for their participation in the study. Furthermore, we are grateful to Miranda van Turennout and Doug Davidson for their contributions in the initial phase of the study and Janine Gossen for her help in recording the stimuli.

\section{References}

Bradley, L., \& Bryant, P. E. (1983). Categorizing sounds and learning to read-A causal connection. Nature, 301, 419-421.

Bryant, P., \& Bradley, L. (1985). Children's reading problems. New York, NY: Blackwell.

Bryant, P. E., MacLean, M., Bradley, L. L., \& Crossland, J. (1990). Rhyme and alliteration, phoneme detection and learning to read. Developmental Psychology, 26, 429-438.

Cardoso-Martins, C. (1994). Rhyme perception: Global or analytical? Journal of Experimental Child Psychology, 57, 26-41.

Carroll, J. M., \& Myers, J. M. (2010). Spoken word classification in children and adults. Journal of Speech, Language and Hearing Research, 54, 127-147.

Carroll, J. M., \& Snowling, M. J. (2001). The effect of global similarity between stimuli on children's judgment of rime and alliteration. Applied Psycholinguistics, 22, 327-342.

Charles-Luce, J., \& Luce, P. A. (1990). Similarity neighbourhoods of words in young children's lexicons. Journal of Child Language, 17, 205-215.

CITO (2009). Screeningsinstrument beginnende geletterdheid (Screening instrument for developping literacy). Arnhem, The Netherlands.

De Cara, B., \& Goswami, U. (2003). Phonological neighbourhood density: Effects in a rhyme awareness task in five-year-old children. Journal of Child Language, 30, 695-710.

de Jong, P. F., \& van der Leij, A. (1999). Specific contributions of phonological abilities to early reading acquisition: Results from a Dutch latent variable longitudinal study. Journal of Educational Psychology, 91, 450-476.

Geudens, A., \& Sandra, D. (2003). Beyond implicit phonological knowledge: No support for an onset-rime structure in children's explicit phonological awareness. Journal of Memory and Language, 49, 157-182.

Geudens, A., Sandra, D., \& Martensen, H. (2005). Rhyming words and onset-rime constituents: An inquiry into the structural breaking points and emergent boundaries in the syllable. Journal of Experimental Child Psychology, 92, 366-387.

Goswami, U. (1999). Causal connections in beginning reading: The importance of rhyme. Journal of Research in Reading, 22, 217-240.

Goswami, U. (2002). Phonology, reading development and dyslexia: A cross-linguistic perspective. Annals of Dyslexia, 52, 1-23.

Goswami, U., \& Bryant, P. (1990). Phonological skills and learning to read. Hillsdale, NJ: Erlbaum.

Hayes, R. A., Slater, A., \& Brown, A. (2000). Infants' ability to categorise on the basis of rhyme. Cognitive Development, 15, 405-419.

Hayes, R. A., Slater, A. M., \& Longmore, C. A. (2009). Rhyming abilities in 9-month-olds: The role of the vowel and coda explored. Cognitive Development, 24, 106-112.

Hulme, C., Hatcher, P. J., Nation, K., Brown, A., Adams, J., \& Stuart, G. (2002). Phoneme awareness is a better predictor of early reading skill than onset-rime awareness. Journal of Experimental Child Psychology, 82, 2-28.

Jarrold, C., Cocksey, J., \& Dockerill, E. (2008). Phonological similarity and lexicality effects in children's verbal short-term memory: Concerns about the interpretation of probed recall data. Quarterly Journal of Experimental Psychology, 61, 324-340.

Johnston, R., Anderson, M., \& Holligon, C. (1996). Knowledge of the alphabet and explicit awareness of phonemes in pre-readers: The nature of the relationship. Reading and Writing: An Interdisciplinary Journal, 8, 217-234. 
Jusczyk, P. W. (1993). From general to language-specific capacities: The WRAPSA model of how speech perception develops. Journal of Phonetics, 21, 3-28.

Kirtley, C., Bryant, P., MacLean, M., \& Bradley, L. (1989). Rhyme, rime, and the onset of reading. Journal of Experimental Child Psychology, 48, 224-245.

Kuhl, P. K., (2004). Early language acquisition: Cracking the speech code. Nature Reviews Neuroscience, 5, 831-843.

Macmillan, B. M. (2002). Rhyme and reading: A critical review of the research methodology. Journal of Research in Reading, 25, 4-42.

Metsala, J. L., \& Walley, A. C. (1998). Spoken vocabulary growth and segmental restructuring of lexical representations: Precursors to phonemic awareness and early reading ability. In J. L. Metsala \& L. C. Ehri (Eds.), Word recognition in beginning literacy (pp. 89-120). Mahwah, NJ, Lawrence Erlbaum Associates.

Nunn, A. (1998). Dutch orthography; A systematic investigation of the spelling of Dutch words. Doctoral dissertation. Nijmegen, The Netherlands, Radboud University.

Schaerlaekens, A., Kohnstamm, D., \& Lejaegere, M. (1999). Streeflijst woordenschat voor 6-jarigen in Nederland en België (Target list vocabulary for 6-year-olds in the Netherlands and Belgium). Lisse, The Netherlands: Swets and Zeitlinger B.V.

Smits, R., Warner, N., McQueen, J. M., \& Cutler, A. (2003). Unfolding of phonetic information over time: A database of Dutch diphone perception. Journal of the Acoustical Society of America, $113,563-574$.

Stadler, M. A., Watson, M., \& Skahan, S. (2007). Rhyming and vocabulary: Effects of lexical restructuring. Communication Disorders Quarterly, 28, 197-205.

Stuart, M. (2005). Phonemic analysis and reading development: some current issues. Journal of Research in Reading, 28, 39-49.

Treiman, R., \& Baron, J. (1981). Segmental analysis ability: Development and relation to reading ability. In G. E. Mackinnon \& T. G. Waller, Reading research: Advances in theory and practice (pp. 159-198). New York, NY, Academic Press, Inc.

Treiman, R., \& Breaux, A. M. (1982). Common phoneme and overall similarity relations among spoken syllables: Their use by children and adults. Journal of Psycholinguistic Research, 11, 569-598.

Treiman, R., \& Kessler, B. (1995). In defense of an onset-rhyme syllable structure for English. Language and Speech, 38, 127-142.

Verhoeven, L. (2011). Screeningsinstrument voor ernstige spraak- en taalmoeilijkheden [Screening instrument for severe speech and language problems]. Arnhem, The Netherlands: CITO.

Vloedgraven, J. M., \& Verhoeven, L. (2007). Screening of phonological awareness in the early elementary grades: An IRT approach. Annals of Dyslexia, 57, 33-50.

Walley, A. (1993). The role of development in children's spoken word recognition and segmentation ability. Developmental Review, 13, 286-350.

Walley, A. C., Metsala, J. L., \& Garlock, V. M. (2003). Spoken vocabulary growth: Its role in the development of phoneme awareness and early reading ability. Reading and Writing: $A n$ Interdisciplinary Journal, 16, 5-20.

Wijnen, F. (1992). Incidental word and sound errors in young speakers. Journal of Memory and Language, 31, 734-755.

Ziegler, J. C., \& Goswami, U. (2005). Reading acquisition, developmental dyslexia, and skilled reading across languages: A psycholinguistic grain size theory. Psychological Bulletin, 131, 3-29.

Zoccolotti, P., De Luca, M., Di Filippo, G., Judica, A., \& Martelli, M. (2009). Reading development in an orthographically regular language: Effects of length, frequency, lexicality and global processing ability. Reading and Writing: An Interdisciplinary Journal, 22, 1053-1079. 


\section{Appendix}

Table Al. Word stimuli used in the rhyme judgement experiment. English translations of the Dutch words are in parentheses

\begin{tabular}{|c|c|c|c|c|c|c|c|c|c|}
\hline \multicolumn{2}{|c|}{$\begin{array}{l}\text { Primes } \\
\text { Rhyme }\end{array}$} & \multicolumn{2}{|c|}{ Consonant overlap } & \multicolumn{2}{|c|}{ Vowel overlap } & \multicolumn{2}{|c|}{ Unrelated } & \multicolumn{2}{|c|}{ Targets } \\
\hline gek & (strange) & bak & (tray) & pet & (cap) & sop & (lather) & bek & (beak) \\
\hline koek & (cookie) & hok & (pen) & poes & (cat) & nies & (sneeze) & hoek & (corner) \\
\hline bil & (buttock) & geel & (yellow) & vis & (fish) & sap & (juice) & gil & (scream) \\
\hline lach & (laugh) & deeg & (dough) & lam & (lamb) & nul & (zero) & dag & (day) \\
\hline zak & (sack) & dik & (thick) & lap & (rag) & pit & (seed) & dak & (roof) \\
\hline life & (sweet) & duif & (pidgeon) & vier & (four) & leeg & (empty) & dief & (thief) \\
\hline bus & (bus) & muis & (mouse) & juf & (teacher) & nek & (nek) & mus & (sparrow \\
\hline ruit & (window) & heet & (hot) & duim & (thumb) & deuk & (dent) & huid & (skin) \\
\hline wip & (seesaw) & kop & (cup) & dit & (this) & bot & (bone) & kip & (chicken) \\
\hline mes & (knife) & los & (loose) & hek & (fence) & pan & (pan) & les & (lesson) \\
\hline top & (top) & pap & (porridge) & $\mathrm{mol}$ & (mole) & kam & (comb) & pop & (doll) \\
\hline naam & (name) & riem & (belt) & kaas & (cheese) & ziek & (sik) & raam & (window) \\
\hline doos & (box) & reus & (giant) & boot & (boat) & tijd & (time) & roos & (rose) \\
\hline jaar & (year) & hier & (here) & maan & (moon) & doof & (deaf) & haar & (hair) \\
\hline lol & (fun) & vel & (sheet) & nog & (still) & pech & (unlucky) & vol & (full) \\
\hline zin & (sentence) & wijn & (wine) & pil & (pill) & kus & (kiss) & win & (win) \\
\hline been & (leg) & gaan & (go) & week & (weak) & duur & (expensive) & geen & (none) \\
\hline map & (folder) & hup & (skip) & jas & (coat) & kok & (cook) & hap & (bite) \\
\hline koel & (cool) & deel & (part) & boer & (farmer) & huis & (house) & doel & (goal) \\
\hline zien & (see) & tuin & (garden) & diep & (deep) & mis & (miss) & tien & (ten) \\
\hline bos & (forest) & vies & (dirty) & zon & (sun) & geit & (goat) & vos & (fox) \\
\hline
\end{tabular}


Table A2. Pseudo-word stimuli used in the rhyme judgement experiment ${ }^{6}$

\begin{tabular}{lcccc}
\hline $\begin{array}{l}\text { Primes } \\
\text { Rhyme }\end{array}$ & Consonant overlap & Vowel overlap & Unrelated & Targets \\
\hline zek & meek & det & pun & mek \\
moek & tuik & voes & nil & toek \\
lil & diel & bis & baf & dil \\
kach & hieg & pam & duk & hag \\
jak & reek & wap & peep & rak \\
bief & haaf & viep & pes & hief \\
tus & has & luf & kech & hus \\
nuit & vijt & luim & deus & vuid \\
mip & buip & mit & gos & bip \\
res & dees & kek & suig & des \\
wop & veep & jol & tiek & vop \\
baam & diem & paas & not & daam \\
goos & wos & hoot & hied & woos \\
laar & ker & paan & zas & kaar \\
zol & guil & bor & nijp & gol \\
din & lon & zig & duuk & lin \\
keen & ran & deek & koom & reen \\
bap & deup & nas & sot & dap \\
loel & hul & doer & mik & hoel \\
bien & gon & jieg & lar & gien \\
zos & pig & mon & keit & pos \\
\hline
\end{tabular}

Table A3. Filler stimuli used in the rhyme judgement experiment. English translations of the Dutch words are in parentheses

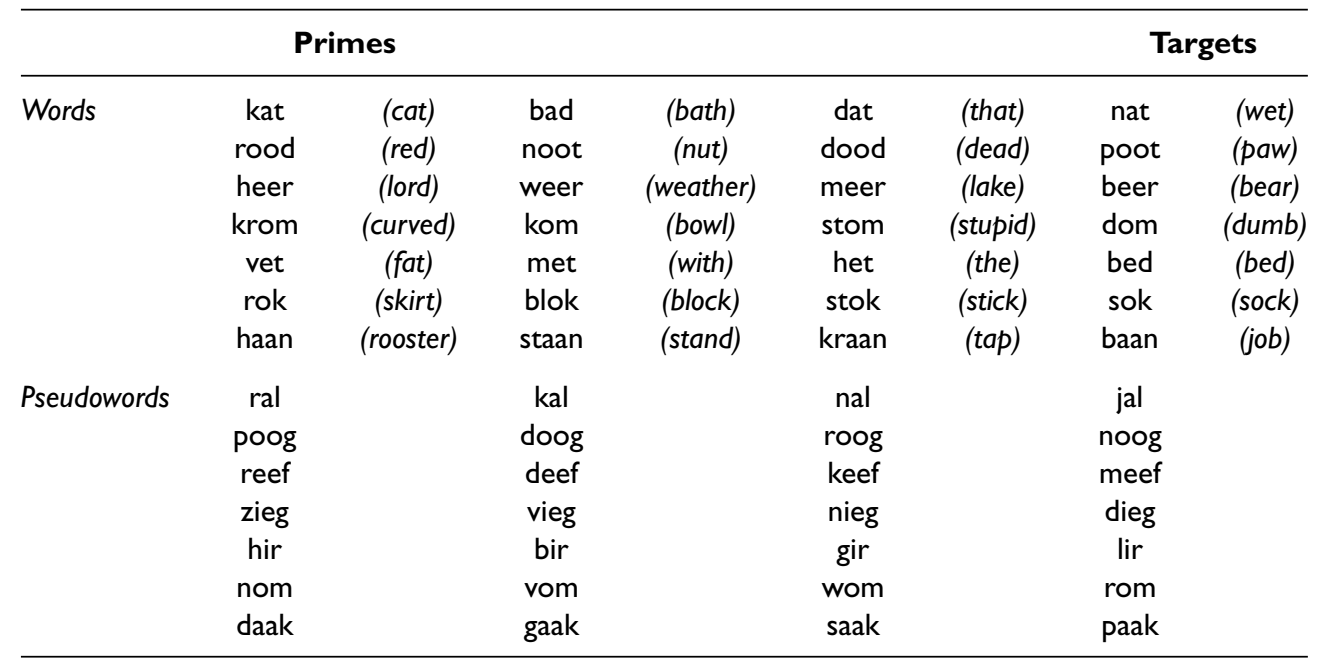

6 'Baf and 'hief' do exist in Dutch language, but are quite rare words and therefore considered pseudo-words in the present study. 